\title{
ON THE EXISTENCE AND CONVERGENCE OF PSEUDOMOMENTS FOR VARIABLES IN THE DOMAIN OF NORMAL ATTRACTION OF AN OPERATOR STABLE DISTRIBUTION
}

\author{
DANIEL CHARLES WEINER
}

(Communicated by Bert E. Fristedt)

\begin{abstract}
Integrals are constructed to replace absolute moments for variables in the domain of normal attraction of an operator stable law. These integrals, called pseudomoments, improve on the geometric information contained in absolute moments. Existence and convergence to appropriate values of these integrals are shown for the variables and their affine normalized sums.
\end{abstract}

1. Introduction. The purpose of this note is to prove the existence and convergence of certain integrals (called pseudomoments) involving weakly convergent affine normalized sums of independent, identically distributed random vectors taking values in a finite-dimensional Euclidean space, when the normalizing operators take on a special form. The existence of these pseudomoments for the common distribution of the summands, and the convergence of the pseudomoments of the affine normalized sums to the corresponding pseudomoments of the limiting distribution, are shown to occur precisely when the latter exist, in the case of a non-Gaussian limit. The case of Gaussian limits is also covered.

The pseudomoments of a distribution (for definitions, see $\$ 2$ ) are designed to reflect the geometry of the distribution more accurately and informatively than do ordinary absolute moments. Since the great utility of affine normalization is its attention to variable decay rates in the probability tails of the one-dimensional projections (marginals) of a distribution, it is clearly an advantage to construct moment-integrals showing similar attention to these marginal tails, as we will see pseudomoments do.

2. Notation, background, and assumptions. Let $(V,\|\cdot\|)$ be a finitedimensional Euclidean space, and let $Z$ be a $V$-valued random vector whose distribution $\mathcal{L}(Z)$ is full, i.e., not supported on any proper hyperplane of $V$. Given a nonsingular linear operator $B$ on $V$ and a number $p>0$, we define the $B$ pseudomoment of order $p$ of $Z$ (equivalently, of $\mathcal{L}(Z)$ ) to be

$$
E(Z, B, p)=\int_{0}^{\infty} P\left(\left\|t^{-B / p} Z\right\| \geq 1\right) d t .
$$

Received by the editors December 13, 1985 and, in revised form, June 13, 1986.

1980 Mathematics Subject Classification (1985 Revision). Primary 60B11, 60F05; Secondary $60 \mathrm{E} 07$.

Key words and phrases. Operator stable laws, moments. pseudomoments, domain of normal attraction, exponents, affine normalization. 
Here, for $s>0$ we define

$$
s^{B}=\exp (B \ln s)=\sum_{K=0}^{\infty} B^{K}(\ln s)^{K} / K !,
$$

so that $t^{B / p}=\left(t^{-1 / p}\right)^{B}$ for $t>0$.

To illustrate the meaning of these integrals, consider the case of $B=\lambda I$ for $\lambda>0$ and $I$ the identity operator on $V$. Then

$$
\begin{aligned}
E(Z, B, p) & =\int_{0}^{\infty} P\left(\|Z\| \geq t^{\lambda / p}\right) d t \\
& =\int_{0}^{\infty} P\left(\|Z\|^{p / \lambda} \geq t\right) d t \\
& =E\|Z\|^{p / \lambda},
\end{aligned}
$$

so that in this case pseudomoments reduce to ordinary absolute moments.

Now let $X, X_{1}, X_{2}, \ldots$ be i.i.d. $V$-valued random vectors with $S_{n}=X_{1}+\cdots+X_{n}$, and assume $X$ is full. When there exist linear operators $T_{n}$ on $V$ and centerings $v_{n} \in V$ such that $\left\{\mathcal{L}\left(T_{n} S_{n}-v_{n}\right)\right\}$ is weakly convergent to a full distribution $\mu$, written $\mathcal{L}\left(T_{n} S_{n}-v_{n}\right) \stackrel{\mathrm{w}}{\rightarrow} \mu$, we say that $X$ (equivalently, $\left.\mathcal{L}(X)\right)$ belongs to the (generalized) domain of attraction of $\mu$ and write $X \in \mathrm{DA}\left(\mu,\left\{T_{n}\right\},\left\{v_{n}\right\}\right)$. Hahn and Klass (1985) have given necessary and sufficient conditions that $X$ belongs to the domain of attraction of a given $\mu$.

By definition, the limiting distribution $\mu$ is operator-stable. We refer to Sharpe (1969) for this definition and the properties of operator-stable laws, but briefly describe the facts we need here.

For $\mu$ full operator-stable on $V$, let $Y, Y_{1}, Y_{2}, \ldots$ be i.i.d. $V$-valued random vectors with common distribution $\mu$. Then there exists a nonsingular linear operator $A$ on $V$, called an exponent for $\mu$ (not necessarily unique, but see Holmes, Hudson, and Mason (1982)), and centerings $y_{n} \in V$ such that for each $n \geq 1$,

$$
\mathcal{L}\left(n^{-A}\left(Y_{1}+\cdots+Y_{n}\right)-y_{n}\right)=\mathcal{L}(Y)=\mu .
$$

(3) is called the stability property of $\mu$. Obviously $Y \in \operatorname{DA}\left(\mu,\left\{n^{-A}\right\},\left\{y_{n}\right\}\right)$, so that the sequence of operators $\left\{n^{-A}\right\}$ plays a special role in the theory of operatorstable laws and their domain of attraction. Sharpe proved that for each choice of $A$, every eigenvalue of $A$ has real part $\geq \frac{1}{2}$.

When the operators $\left\{T_{n}\right\}$ used to normalize the sums $S_{n}$ can be chosen in the special form $T_{n}=n^{-A}$ for some exponent $A$ for $\mu$ (i.e., $\left.X \in \operatorname{DA}\left(\mu,\left\{n^{-A}\right\},\left\{v_{n}\right\}\right)\right)$, we say $X$ belongs to the domain of normal attraction of $\mu$, and write

$$
X \in \operatorname{DNA}\left(\mu, A,\left\{v_{n}\right\}\right) .
$$

Now $X$ will belong to the DNA of $\mu$ regardless of choice of exponent $A$ for $\mu$ : only the centerings $\left\{v_{n}\right\}$ will be affected (Jurek (1980)). Thus we will occasionally write $X \in \mathrm{DNA}(\mu)$. For more on domains of normal attraction, see Hudson, Mason, and Veeh (1983), and Jurek $(1980,1982)$, and Hahn and Hudson (1987).

When $X \in \operatorname{DNA}\left(\mu, A,\left\{v_{n}\right\}\right)$, it is clear that the natural integrals to consider are the pseudomoments $E(X, A, p)$ and $E\left(n^{-A} S_{n}-v_{n}, A, p\right)$. Part of the motivation of our theorem is the following standard fact about ordinary stable laws and variables in their domains of normal attraction (see, e.g., Feller (1971)): 
If $\mu$ is an ordinary stable law on $V$ with index $0<\alpha<2$, and $X \in \operatorname{DNA}(\mu)$, then $E\|X\|^{p}<\infty$ if $p<\alpha$ and $E\|X\|^{p}=\infty$ if $p \geq \alpha$.

For such $\mu$, we can take as exponent $A=I / \alpha$ (see (1)) and then from (2) we see $E(X, A, p)=E\|X\|^{\alpha p}$, which is finite if and only if $p<1$.

3. Results and proofs. We are ready to state our results.

THEOREM. Let $X \in \mathrm{DNA}(\mu)$ and $\mathcal{L}(Y)=\mu$. Then, for each exponent $A$ for $\mu$,

(a) $E(Y, A, p)<\infty$ for $p<1$,

(b) if $\mu$ is Gaussian, $E(Y, A, p)<\infty$ for all $p$, while if $\mu$ is not Gaussian, $E(Y, A, p)=\infty$ for $p \geq 1$,

(c) $E(X, A, p)<\infty$ for $p<1$,

(d) if $\mu$ is Gaussian, $E(X, A, 1)<\infty$, while if $\mu$ is not Gaussian, $E(X, A, p)=\infty$ for $p \geq 1$,

(e) if $X \in \operatorname{DNA}\left(\mu, A,\left\{v_{n}\right\}\right)$,

$$
\lim _{n \rightarrow \infty} E\left(n^{-A} S_{n}-v_{n}, A, p\right)=E(Y, A, p)
$$

for $p<1$, while if $\mu$ is Gaussian, equality in (4) also holds for $p=1$.

Before proving the Theorem, we remark that these existence and convergence results extend Theorem 3 in Hudson, Veeh, and Weiner (1987) from absolute moments (in the general domain of attraction) to pseudomoments (but only in the domain of normal attraction). The natural conjecture is that our theorem (suitably modified when $p=1$ ) remains true for general domains, but we have been unable to achieve this result.

We also observe that the existence of a so-called invariant norm $\|\cdot\|$ for $\mu$ on $V$ (see Hudson, Jurek, and Veeh (1986)) can be used to establish, in this norm, that the value of $E(Y, A, p)$ is independent of choice of exponent $A$ for $\mu$, for each $p>0$. However, even in this norm the value (though not the finiteness) of $E(X, A, p)$ depends on $A$, so we do not pursue the matter here.

PROOF OF THE THEOREM. The proof of the Theorem rests on the following adaptation of the uniform integrability criterion for convergence of moments (cf. Chung (1974, Theorem 4.5.2)):

LEMMA. Assume $Z, Z_{1}, Z_{2}, \ldots$ are full $V$-valued r.v.'s, and that $\mathcal{L}\left(Z_{n}\right) \stackrel{\mathrm{w}}{\rightarrow} \mathcal{L}(Z)$, where $\mathcal{L}(Z)$ is absolutely continuous with respect to Lebesgue measure. Let $B$ be a nonsingular linear operator on $V$. If for some $q>0$ we have $C=\sup _{n} E\left(Z_{n}, B, q\right)$ $<\infty$, then for each $p<q$ such that $E(Z, B, p)<\infty$, we have

$$
\lim _{n \rightarrow \infty} E\left(Z_{n}, B, p\right)=E(Z, B, p) \text {. }
$$

ProOF OF THE LEMMA. For every $t>0$ such that $P\left(\left\|t^{-B / p} Z\right\|=1\right)=0$, we have

$$
g_{n}(t)=P\left(\left\|t^{-B / p} Z_{n}\right\| \geq 1\right) \rightarrow g(t)=P\left(\left\|t^{-B / p} Z\right\| \geq 1\right) .
$$

Now the linear map $t^{-B / p}: V \rightarrow V$ is nonsingular, so if $S=\{x \in V:\|x\|=1\}$, then $P\left(\left\|t^{-B / p} Z\right\|=1\right)=P\left(Z \in t^{B / p}(S)\right)=0$, since $\mathcal{L}(Z)$ is absolutely continuous with respect to Lebesgue measure on $V$. Thus $g_{n}(t) \rightarrow g(t)$ for each $t>0$. 
Given $R>0$, an application of the Bounded Convergence Theorem (since $g_{n}(t) \leq$ 1 for all $t>0$ ) gives

$$
\lim _{n \rightarrow \infty} \int_{0}^{R} g_{n}(t) d t=\int_{0}^{R} g(t) d t .
$$

For any $R>0$, the change of variables $t^{q}=u^{p}$ gives

$$
\begin{aligned}
\int_{R}^{\infty} g_{n}(t) d t & =\frac{p}{q} \int_{R^{q / p}}^{\infty} P\left(\left\|u^{-B / q} Z_{n}\right\| \geq 1\right) \frac{d u}{u^{(q-p) / q}} \\
& \leq \frac{p}{q R^{(q-p) / p}} \int_{0}^{\infty} P\left(\left\|u^{-B / q} Z_{n}\right\| \geq 1\right) d u \\
& \leq \frac{C p}{q R^{(q-p) / p}}
\end{aligned}
$$

uniformly in $n$. Since $p<q, \lim _{R \rightarrow \infty} \int_{R}^{\infty} g_{n}(t) d t=0$ uniformly in $n$.

But, as $E(Z, B, p)<\infty$, we also have $\lim _{R \rightarrow \infty} \int_{R}^{\infty} g(t) d t=0$.

Thus given $\varepsilon>0$, choose $R$ so large that $\int_{R}^{\infty} g(t) d t<\varepsilon / 3$ and $\sup _{n} \int_{R}^{\infty} g_{n}(t) d t<$ $\varepsilon / 3$. For this $R$ choose $N$ so large that $n \geq N$ implies

$$
\left|\int_{0}^{R} g_{n}(t) d t-\int_{0}^{R} g(t) d t\right|<\frac{\varepsilon}{3} \text {. }
$$

Then for $n \geq N$,

$$
\begin{aligned}
\left|E\left(Z_{n}, B, p\right)-E(Z, B, p)\right| & =\left|\int_{0}^{\infty}\left(g_{n}(t)-g(t)\right) d t\right| \\
& \leq\left|\int_{0}^{R}\left(g_{n}-g\right)\right|+\int_{R}^{\infty} g_{n}+\int_{R}^{\infty} g<\frac{\varepsilon}{3}+\frac{\varepsilon}{3}+\frac{\varepsilon}{3}=\varepsilon
\end{aligned}
$$

whence $E\left(Z_{n}, B, p\right) \rightarrow E(Z, B, p)$ as asserted.

To prove the Theorem, we modify an argument of de Acosta (1979 and 1980); as compared to the argument in Hudson, Veeh, and Weiner (1987), the work here is simplified because "regular variation" of the operators $\left\{n^{-A}\right\}$ for normal attraction is built in; no convergence-of-types arguments are required to see, for example, that for every $m$, we have $(m n)^{-A} n^{A} \rightarrow m^{-A}$ as $n \rightarrow \infty$.

Since $\mu$ is infinitely divisible, by classical results we can write the characteristic function $\hat{\mu}$ of $\mu$ as

$$
\hat{\mu}(t)=\exp \left(i(a, t)-\frac{1}{2}(t, \Sigma t)+\int\left(e^{i(t, x)}-1-i \alpha(t, x)\right) d M(x)\right),
$$

where $(\cdot, \cdot)$ is an inner product generating $\|\cdot\|$ and for some $\delta>0, \alpha(t, x)=$ $\min (\delta,\|x\|)(t, x) /\|x\| ; M$ is the Lévy measure on $V \backslash\{0\}$ associated with $\mu, a \in V$, and $\Sigma$ is a linear operator on $V$.

First let us dispose of the purely Gaussian case, i.e., $M \equiv 0$. Then one admissible exponent for $\mu$ is $A=\frac{1}{2} I$, so that pseudomoments reduce to ordinary moments for this choice of exponent. Our assertions are well known in this case; it is easy to check that other choices will not affect the results, and we refer to Hudson, Mason, and Veeh (1983) for the necessary details on exponents for Gaussian laws.

Thus we assume for the remainder of the proof that $M$ in (5) is not the zero measure on $V \backslash\{0\}$. 
Fix an exponent $A$ for $\mu$.

The fact (communicated by Sharpe to Hudson (1980)) that operator-stable laws have Lebesgue densities will allow us to apply the Lemma. We will also require this consequence of the stability property (3) proved by Sharpe (1969): For each $t>0$ and Borel set $B \subset V \backslash\{0\}$,

$$
M\left\{x \in V: t^{-A} x \in B\right\}=t^{-1} M(B) .
$$

Now let $\varepsilon>0, B_{\varepsilon}=\{x \in V:\|x\| \geq \varepsilon\}$. Then (6) implies $t M\left(\left\{x \in V:\left\|t^{-A} x\right\| \geq\right.\right.$ $\varepsilon\}=M\left(B_{\varepsilon}\right)$ for all $t>0$. It is an easy consequence of the nonsingularity of $A$ that $M\left(B_{\varepsilon}\right)>0$ for every $\varepsilon>0$, lest $M \equiv 0$.

As each eigenvalue of $A$ has strictly positive real part, the two triangular arrays $\left\{n^{-A} X_{j}: j \leq n, n \geq 1\right\}$ and $\left\{n^{-A} Y_{j}: j \leq n, n \geq 1\right\}$, the row sums for each of which are (shift) convergent in distribution to $\mu$, are infinitesimal. Assume for the moment that $\|\cdot\|$ is the "invariant norm" of Hudson, Jurek, and Veeh (1986). Application of their Proposition 3 (in particular, (iv)) and Theorem 6 show that, in their norm, $M\left\{x \in V:\left\|t^{-A}\right\|=\varepsilon\right\}=0$ for each $\varepsilon>0$. Thus the Central Limit Theorem (see, e.g., Araujo and Giné (1980)) yields (again in their norm) that for every $\varepsilon>0$,

$$
\infty>\lim _{n \rightarrow \infty} n P\left(\left\|n^{-A} X\right\| \geq \varepsilon\right)=\lim _{n \rightarrow \infty} n P\left(\left\|n^{-A} Y\right\| \geq \varepsilon\right)=M\left(B_{\varepsilon}\right)>0 .
$$

Now the compatibility of all norms on the finite-dimensional vector space $V$, together with an easy continuity argument, give us, in the original norn, (8)

$$
\begin{aligned}
& 0<\liminf _{t \rightarrow \infty} t P\left(\left\|t^{-A} X\right\| \geq \varepsilon\right) \leq \limsup _{t \rightarrow \infty} t P\left(\left\|t^{-A} X\right\| \geq \varepsilon\right)<\infty \\
& 0<\liminf _{t \rightarrow \infty} t P\left(\left\|t^{-A} Y\right\| \geq \varepsilon\right) \leq \limsup _{t \rightarrow \infty} t P\left(\left\|t^{-A} Y\right\| \geq \varepsilon\right)<\infty \quad \text { for each } \varepsilon>0 .
\end{aligned}
$$

Now (a) through (d) are immediate from (8), upon replacing $t$ by $t^{1 / p}$, and it remains only to show the convergence in (4).

First assume $X$ is symmetric. We now have $\mathcal{L}\left(n^{-A} S_{n}\right) \stackrel{\mathrm{w}}{\rightarrow} \mu$.

By one of Lévy's maximal inequalities (see, e.g., Araujo and Giné (1980), Theorem 3.2.6), for each $R>0$ and integer $m \geq 1$ we have (letting $S_{0}=0$ )

$$
\begin{aligned}
2 P\left(\left\|(m n)^{-A} S_{n m}\right\|>R\right) & \geq P\left(\max _{1 \leq j \leq m}\left\|(m n)^{-A}\left(S_{n j}-S_{n(j-1)}\right)\right\|>R\right) \\
& =1-P\left(\left\|(m n)^{-A} S_{n}\right\| \leq R\right)^{m} \\
& =1-\left(1-P\left(\left\|(m n)^{-A} S_{n}\right\|>R\right)\right)^{m},
\end{aligned}
$$

using the i.i.d. assumption.

Now $\left\{\mathcal{L}\left((m n)^{-A} S_{n m}\right): m \geq 1, n \geq 1\right\}$ is tight, so given $\varepsilon>0$ there exists $R>0$ such that $\sup _{m, n} P\left(\left\|(m n)^{-A} S_{n m}\right\| \geq R\right)<\varepsilon / 2$. Choose $\varepsilon$ small enough that there exists $B>0$ such that $b<\varepsilon$ implies $m\left(1-(1-b)^{1 / m}\right) \leq B$ for all $m \geq 1$.

We obtain from (9) that for sufficiently large $R$,

$$
\sup _{m, n} m P\left(\left\|(m n)^{-A} S_{n}\right\| \geq R\right) \leq B<\infty .
$$


Now (10) implies that for sufficiently large $t_{0}$,

$$
C=\sup _{t \geq t_{0}} \sup _{n} t P\left(\left\|t^{-A} n^{-A} S_{n}\right\| \geq 1\right)<\infty .
$$

To see this, let $m=\alpha t$ in (10). The inequality $\|T x\| \geq\|x\| /\left\|T^{-1}\right\|$ for $x \in V$ and linear $T$ on $V$ applies, together with another easy continuity argument, to give

$$
\begin{aligned}
B / \alpha & \geq \sup _{t \geq 1 / \alpha} \sup _{n} t P\left(\left\|\alpha^{-A} t^{-A} n^{-A} S_{n}\right\| \geq R\right) \\
& \geq \sup _{t \geq 1 / \alpha} \sup _{n} t P\left(\left\|t^{-A} n^{-A} S_{n}\right\| \geq R\left\|\alpha^{A}\right\|\right) \\
& \geq \sup _{t \geq 1 / \alpha} \sup _{n} t P\left(\left\|t^{-A} n^{-A} S_{n}\right\| \geq 1\right)
\end{aligned}
$$

for $\alpha$ sufficiently small that $R\left\|\alpha^{A}\right\| \leq 1$. (That $\lim _{\alpha \rightarrow 0}\left\|\alpha^{A}\right\|=0$ follows from the fact in Sharpe (1969) that every eigenvalue of $A$ has real part strictly positive.) Putting $t_{0}=1 / \alpha$ yields (11).

Now fix $p<q<1$. Since $E(Y, A, p)<\infty$, to apply the lemma we must estimate

$$
\begin{aligned}
E\left(n^{-A} S_{n}, A, q\right) & =\int_{0}^{\infty} P\left(\left\|t^{-A / q}\left(n^{-A} S_{n}\right)\right\| \geq 1\right) d t \\
& =\int_{0}^{\infty} t^{1 / q} P\left(\left\|\left(t^{1 / q}\right)^{-A}\left(n^{-A} S_{n}\right)\right\| \geq 1\right) \frac{d t}{t^{1 / q}} \\
& \leq t_{0}^{q}+C \int_{t_{0}^{q}}^{\infty} \frac{d t}{t^{1 / q}}=t_{0}^{q}+\frac{C q}{q-1} t_{0}^{(q-1) / q}
\end{aligned}
$$

uniformly in $n$, using (11).

Thus the lemma gives (4) and it remains only to desymmetrize.

If $X$ is not symmetric, assume $\mathcal{L}\left(n^{-A} S_{n}-v_{n}\right) \stackrel{\text { w }}{\rightarrow} \mu$.

Let $X_{1}^{\prime}, X_{2}^{\prime}, \ldots$ be i.i.d., independent of $X_{1}, X_{2}, \ldots$, and with common distribution $\mathcal{L}(X)$. Put $S_{n}^{\prime}=X_{1}^{\prime}+\cdots+X_{n}^{\prime}$. Let $Y^{\prime}$ be independent of $Y$, with $\mathcal{L}\left(Y^{\prime}\right)=\mathcal{L}(Y)=\mu$.

Now $Y-Y^{\prime}$ is operator stable with $A$ as an exponent, as is easy to check, and thus for every $p<1, E\left(Y-Y^{\prime}, A, p\right)<\infty$. We also have $\mathcal{L}\left(n^{-A}\left(S_{n}-S_{n}^{\prime}\right)\right) \stackrel{\mathrm{w}}{\rightarrow} \mathcal{L}\left(Y-Y^{\prime}\right)$, so by the symmetric case, for each $q<1$ we obtain

$$
\lim _{n \rightarrow \infty} E\left(n^{-A}\left(S_{n}-S_{n}^{\prime}\right), A, q\right)=E\left(Y-Y^{\prime}, A, q\right) .
$$

Once again we seek to apply the Lemma: Fix $p<q<1$. We need only show $\left.\sup _{n} E\left(n^{-A} S_{n}-v_{n}\right) A, q\right)<\infty$.

Let $D=\sup _{n} E\left(n^{-A}\left(S_{n}-S_{n}^{\prime}\right), A, q\right)$, and define $g_{n}(y)=E\left(n^{-A} S_{n}-v_{n}-y, A, q\right)$. By Fubini's Theorem,

$$
\int g_{n}(y) d \mathcal{L}\left(n^{-A} S_{n}-v_{n}\right)(y)=E\left(n^{-A}\left(S_{n}-S_{n}^{\prime}\right), A, q\right) \leq D
$$

Choose $R>0$ so large that $\left.\sup _{n} P\left(\| n^{-A} S_{n}-v_{n}\right) \geq R\right)<\frac{1}{2}$, and let $B(R)=$ $\{x \in V:\|x\| \leq R\}$. Then for all $n$ sufficiently large, there exists $y_{n} \in B(R)$ such 
that $g_{n}\left(y_{n}\right) \leq 3 D$, for suppose not. Then

$$
\begin{aligned}
D & \geq \liminf _{n \rightarrow \infty} \int_{B(R)} g_{n}(y) d \mathcal{L}\left(n^{-A} S_{n}-v_{n}\right)(y) \\
& \geq 3 D \liminf _{n \rightarrow \infty} P\left(n^{-A} S_{n}-v_{n} \in B(R)\right) \\
& \geq 3 D\left(\frac{1}{2}\right)=\frac{3}{2} D,
\end{aligned}
$$

a contradiction since $Y$ full implies $E(Y, A, q)>0$ (and thus $D>0$ ).

So choose $y_{n} \in B(R)$ with $g_{n}\left(y_{n}\right) \leq 3 D$. Since $\left\{y_{n}\right\}$ is bounded and the eigenvalues of $A$ all have strictly positive real part, we know $\lim _{t \rightarrow \infty}\left\|t^{-A} y_{n}\right\|=0$ uniformly in $n$. But then

$$
\begin{aligned}
E\left(n^{-A} S_{n}-v_{n}, A, q\right)= & \int_{0}^{\infty} P\left(\left\|t^{-A / q}\left(n^{-A} S_{n}-v_{n}\right)\right\| \geq 1\right) d t \\
\leq & \int_{0}^{\infty} P\left(\left\|t^{-A / q}\left(n^{-A} S_{n}-v_{n}-y_{n}\right)\right\| \geq \frac{1}{2}\right) d t \\
& +\int_{0}^{\infty} P\left(\left\|t^{-A / q} y_{n}\right\| \geq \frac{1}{2}\right) d t
\end{aligned}
$$

The last term in (13) is uniformly bounded, by the previous remark; the change of variables $t=\alpha u$ in the first term, and an argument similar to that used to show (10) implies (11) earlier, lead to a bound of some constant (independent on $n$ ) times $g_{n}\left(y_{n}\right)$; since $g_{n}\left(y_{n}\right) \leq 3 D$, the proof is complete.

REMARK. The author wishes to thank the referee for a very careful reading of this manuscript, and especially for helping us clarify (7) and (8).

\section{BIBLIOGRAPHY}

A. de Acosta, (1979) Convergence of moments and related functionals in the general central limit theorem in Banach spaces, Z. Wahrsch. Verw. Gebiete 48, 213-231.

, (1980) Exponential moments of vector valued random series and triangular arrays, Ann. Probab. 2, 381-389.

A. Araujo and E. Giné, (1980) The central limit theorem for real and Banach valued random variables, Wiley, New York.

K. Chung, (1974) A course in probability theory, Academic Press, New York.

W. Feller, (1971) An introduction to probability theory and its applications, Vol. II, Wiley, New York.

M. Hahn and W. Hudson, (1987) Operator stable laws: Series representations and domains of normal attraction, Preprint.

M. Hahn and M. Klass, (1985) Affine normability of partial sums of i.i.d. random vectors: $A$ characterization, Z. Wahrsch. Verw. Gebiete 69, 479-505.

J. Holmes, W. Hudson, and D. Mason, (1982) Operator-stable laws: Multiple exponents and elliptical symmetry, Ann. Probab. 3, 602-612.

W. Hudson, (1980) Operator-stable distributions and stable marginals, J. Multivariate Anal. 1, 26-37.

W. Hudson, Z. Jurek, and J. Veeh, (1986) The symmetry group and exponents of operator stable probability measures, Ann. Probab. 3, 1014-1023.

W. Hudson and D. Mason, (1981) Operator-stable distribution on $\mathbf{R}^{2}$ with multiple exponents, Ann. Probab. 3, 482-489.

W. Hudson, D. Mason, and J. Veeh, (1983) The domain of normal attraction of an operator-stable law, Ann. Probab. 1, 178-184. 
W. Hudson, J. Veeh, and D. Weiner, (1987) Moments of distributions attracted to operator stable laws, J. Multivariate Anal. (to appear).

Z. Jurek, (1980) Domains of normal attraction of operator-stable measure on Euclidean spaces, Bull. Acad. Polon. Sci. 17, 397-409.

(1982) Domains of nornal attraction for $G$-stable measures on $\mathbf{R}^{d}$, Theory Probab. Appl. 27, 419-424.

J. Kucharczak, (1976) Remark on operator-stable measures, Colloq. Math. 34, 109-119.

M. Sharpe, (1969) Operator-stable probability distributions on vector groups, Trans. Amer. Math. Soc. 136, 51-65.

Department of Mathematics and Statistics, University of Nebraska, LinCOLN, NEBRASKA 68588-0323

Current address: Department of Mathematics, Boston University, Boston, Massachusetts 02215 